\title{
HSF1 Attenuates LPS-Induced Acute Lung Injury in Mice by Suppressing Macrophage Infiltration
}

\author{
Tao Li, ${ }^{1,2}$ Gui Xiao, ${ }^{1,3}$ Sipin Tan, ${ }^{1}$ Xueyan Shi, ${ }^{1}$ Leijing Yin, ${ }^{1}$ Chuyi Tan, ${ }^{1}$ Jia Gu, ${ }^{1}$ \\ Yanjuan Liu, ${ }^{1}$ Huafei Deng, ${ }^{1}$ Ke Liu, ${ }^{1}$ Meidong Liu, ${ }^{1}$ Huali Zhang $\mathbb{D}^{1},{ }^{1}$ and Xianzhong Xiao ${ }^{1}{ }^{1}$ \\ ${ }^{1}$ Key Laboratory of Sepsis Translational Medicine of Hunan, Department of Pathophysiology, Xiangya School of Medicine, \\ Central South University, Changsha, Hunan 410008, China \\ ${ }^{2}$ Department of Pathophysiology, Medical College of Jiaying University, Meizhou, Guangdong 514031, China \\ ${ }^{3}$ Department of Nursing, Hainan Medical University, Haikou, Hainan 571199, China
}

Correspondence should be addressed to Huali Zhang; zhanghuali@csu.edu.cn and Xianzhong Xiao; xiaoxianzhong@csu.edu.cn

Received 2 April 2020; Revised 21 June 2020; Accepted 1 December 2020; Published 18 December 2020

Academic Editor: Manjit Singh Rana

Copyright (C) 2020 Tao Li et al. This is an open access article distributed under the Creative Commons Attribution License, which permits unrestricted use, distribution, and reproduction in any medium, provided the original work is properly cited.

Heat shock factor 1 (HSF1) is a transcription factor involved in the heat shock response and other biological processes. We have unveiled here an important role of HSF1 in acute lung injury (ALI). HSF1 knockout mice were used as a model of lipopolysaccharide- (LPS-) induced ALI. Lung damage was aggravated, and macrophage infiltration increased significantly in the bronchoalveolar lavage fluid (BALF) and lung tissue of $\mathrm{HSF}^{--}$mice compared with the damage observed in $\mathrm{HSF}^{+/+}$mice. Upon LPS stimulation, $\mathrm{HSF}^{-/-}$mice showed higher levels of monocyte chemoattractant protein-1 (MCP-1) in the serum, BALF, and lung tissue and increased the expression of MCP-1 and chemokine (C-C motif) receptor 2 (CCR2) on the surface of macrophages compared with those in $\mathrm{HSF}^{+/+}$. Electrophoretic mobility shift assays (EMSA) and dual luciferase reporter assays revealed that HSF1 could directly bind to heat shock elements (HSE) in the promoter regions of MCP-1 and its receptor CCR2, thereby inhibiting the expression of both genes. We concluded that HSF1 attenuated LPS-induced ALI in mice by directly suppressing the transcription of MCP-1/CCR2, which in turn reduced macrophage infiltration.

\section{Introduction}

Acute lung injury is a type of acute and progressive hypoxic respiratory failure, where noncardiac pathogenic factors inside and outside the lungs cause damage to the alveolar and capillary endothelia, increasing the permeability of alveolar-capillary membrane. If the condition is not controlled, it will progress to acute respiratory distress syndrome (ARDS) (1) eventually leading to death. The pathogenesis of ALI is complex and has not been fully elucidated yet. Current studies indicate that inflammation is an important mechanism of ALI (2).

HSF1 is a transcription factor implicated in the heat shock response; it regulates the transcription of heat shock proteins such as Hsp27, Hsp60, and Hsp70, which play an important cytoprotective role in lung inflammation and injury (3-6). Our previous studies showed that HSF1 has a protective effect on LPS-induced multiorgan dysfunction syndrome $(7,8)$. Furthermore, HSF1 reduced leukocyte infiltration into the lungs and decreased the production of several inflammatory mediators, thereby attenuating inflammatory responses and exhibiting a protective effect on endotoxemia caused by LPS. However, the underlying mechanisms by which HSF1 alleviates ALI need to be further explored.

Macrophages are important effector cells in the pathogenesis of ALI. It has been reported that macrophage activation and migration are closely related to the severity of ALI (9). However, the effect of HSF1 on macrophages in ALI remains unclear. This study is aimed at exploring the effect of HSF1 on macrophages in ALI, by assessing the role of HSF1 in regulating chemokine expression. To the best of our knowledge, this is the first report showing that HSF1 
alleviates LPS-induced macrophage infiltration and ALI in mice, by downregulating macrophage-related chemokines.

\section{Materials and Methods}

2.1. Animals. HSF1 knockout $\left(\mathrm{HSF}^{-/-}\right)$and wild-type $\left(\mathrm{HSF}^{+/+}\right)$mice were gifts from Dr. Ivor J. Benjamin (Medical College of Wisconsin, Milwaukee city, Wisconsin, USA) and have been described elsewhere (10). HSF1 mice were created using homologous recombination with a gene-targeting vector in embryonic stem cells as described by McMillan et al. (11). After mating HSF1 heterozygous female mice $\left(\right.$ oHSF $^{+/-}$) with HSF1 heterozygous male mice $\left({ }^{\top} \mathrm{HSF}^{+/-}\right.$), offspring with three genotypes was obtained: wild type, heterozygous type, and homozygous type. 3 weeks after birth, young mice were weaned and kept in separate cages. At the fourth week, mouse were genotyped using DNA from tail to identify qualified HSF1 knockout homozygous ( $\mathrm{HSF}^{-/-}$) and wild-type $\left(\mathrm{HSF}^{+/+}\right)$mice for the experiments. Protocols for animal breeding and experiments were previously approved by the Institutional Animal Care and Use Committee of Central South University (Hunan, China) under license number 2018sydw0378 (approval date: 25 Nov., 2018).

2.2. Acute Lung Injury Model. Age- and sex-matched HSF1 ${ }^{-/-}$ and $\mathrm{HSF}^{+/+}$mice (males, 4-5 months old, 20-25 g) were randomly divided into the $\mathrm{HSF}^{+/+}+$normal saline (NS) group, $\mathrm{HSF}^{+/+}+\mathrm{LPS}$ (E. coli 0111:B4, Sigma, USA) group, HSF1 ${ }^{1-}+\mathrm{NS}$ group, and $\mathrm{HSF}^{-/-}+\mathrm{LPS}$ group. Mice were anesthetized with $5 \%$ chloral hydrate $(0.01 \mathrm{ml} / \mathrm{g}$, intraperitoneally) and $2 \%$ isoflurane (inhalation). Then, LPS $(1 \mathrm{mg} / \mathrm{ml})$ was instilled into the trachea at a dose of $3 \mathrm{mg} / \mathrm{kg}$ (12) in $\mathrm{HSF}^{+/+}+\mathrm{LPS}$ and $\mathrm{HSF}^{-/-}+\mathrm{LPS}$ groups. $\mathrm{HSF}^{+/+}+\mathrm{NS}$ and $\mathrm{HSF}^{-1-}+\mathrm{NS}$ groups were instilled with normal saline using the same dose and procedure as for LPS. Treatments were evaluated after $12 \mathrm{~h}, 24 \mathrm{~h}$, and $36 \mathrm{~h}$. In this experiment, pain and distress assessment of mice was conducted from postoperative to euthanasia. The method is as follows: mice were placed in a small transparent observation room $(10 \times 10 \times 10 \mathrm{~cm})$ for monitoring, and each mouse was observed for 3-4 minutes. According to GAP scores (13), pain indicators such as activity, posture, breathing pattern, coat condition, and relation to other mice were recorded, respectively. A blind observer assessed each mouse at 1, 6, 12,24 , and $36 \mathrm{~h}$ after the operation according to the time of sample collection. Mice were assigned a score of 0 (normal) or 1 (abnormal) for each parameter. Scores were recorded based on changes in pain indicators and then aggregated to get a composite score for each mouse. Mice in the LPStreated group showed symptoms such as deeper breathing, decrease in movement, hair erect, back arch, reduced consumption of feed and water, and increased body temperature after using LPS. Therefore, in order to exclude the interference caused by LPS, we strictly observed differences between the LPS-treated group and the saline group and then scored the mice in the LPS-treated group.

2.3. Lung Wet/Dry Weight (W/D) Ratio. Pulmonary edema in treated groups was assessed using the lung wet/dry weight ratio. Mice were killed, and the lungs were removed and weighed (wet weight). Lungs were heated at $80^{\circ} \mathrm{C}$ for $24 \mathrm{~h}$ to obtain the dry weight and calculate the $\mathrm{W} / \mathrm{D}$ ratio.

2.4. Extraction of Bronchoalveolar Lavage Fluid. BALF was collected as previously described (14). Mice were sacrificed by cervical dislocation. The skin of the neck was cut off to expose the trachea, and a small oblique incision was made at the telecentric end of the trachea using ophthalmic scissors. Then, a trocar was inserted into the trachea to wash mice lungs thrice by flushing $1 \mathrm{ml}$ of cold sterile phosphatebuffered saline (PBS) per wash. The collected lavage fluid was centrifuged at $250 \times g$ for 15 minutes at $4^{\circ} \mathrm{C}$. The cell pellet was resuspended in PBS for flow cytometry detection. The supernatant was stored at $-80^{\circ} \mathrm{C}$ until further use.

2.5. Enzyme-Linked Immunosorbent Assay (ELISA). Supernatants from lung tissue homogenate, BALF, and serum were collected to measure the levels of MCP-1 using an ELISA kit (MJE00B, RD, USA) according to the manufacturer's instructions.

2.6. Immunofluorescence. Immunofluorescence analysis was performed as previously published (15). Briefly, ALI model mice were sacrificed at different time points. The left lung was removed immediately after death and fixed in $4 \%$ paraformaldehyde for $24 \mathrm{~h}$. After embedding in paraffin, the lung tissue was cut into $4 \mu \mathrm{m}$ thick sections. For antigen retrieval, sections were placed in sodium citrate buffer and heated in a microwave at $100^{\circ} \mathrm{C}$ for $15 \mathrm{~min}$, then incubated with $10 \%$ bovine serum albumin (BSA) in PBS for $2 \mathrm{~h}$ at room temperature to block nonspecific binding sites. Subsequently, sections were incubated with murine macrophage-monocyte specific monoclonal antibody(anti-F4/80) (1:200, Santa Cruz, USA) and C-C chemokine receptor type 2 monoclonal antibody (anti-CCR2) (1:100, Abcam, USA) overnight at $4^{\circ} \mathrm{C}$, followed by incubation with horseradish peroxidase secondary antibodies (1:50, ABclonal, Wuhan) for $1 \mathrm{~h}$ at $37^{\circ} \mathrm{C}$ under darkness. After washing 4 times with PBS, samples were treated with sealing liquid. Images were obtained using Panoramic 250/MIDI (Hungary).

2.7. Flow Cytometry. Cells collected from BALF and prepared for flow cytometric analysis as previously reported (16). Cells were suspended in $1 \mathrm{ml}$ of $1 \times$ red blood cell lysis buffer (BD Biosciences, USA) for 5 minutes, then centrifuged at $250 \times$ $g$ for $5 \mathrm{~min}$. The supernatant containing BALF cells was resuspended in $100 \mu \mathrm{l}$ PBS, then blocked with $0.5 \mu \mathrm{l}$ of purified rat anti-mouse CD16/CD32 antibody (BD Biosciences, USA) for $10 \mathrm{~min}$ at $4^{\circ} \mathrm{C}$. BALF cells were stained with fluorescent-conjugated antibodies: PerCP-Cy ${ }^{\mathrm{TM}} 5.5$ rat antimouse CD45 antibody (0.5 $\mu \mathrm{l}$, BD Biosciences, USA), PE anti-mouse F4/80 antibody ( $0.5 \mu \mathrm{l}$, BD Biosciences, USA), and Alexa Fluor 647 anti-mouse CCR2 (0.5 $\mu \mathrm{l}$, Biolegend, USA) for $20 \mathrm{~min}$ at $4^{\circ} \mathrm{C}$ under darkness, then centrifuged at $250 \times g$ for $5 \mathrm{~min}$. The supernatant was fixed with $100 \mu \mathrm{l}$ of $2 \%$ paraformaldehyde at $4^{\circ} \mathrm{C}$ for $10 \mathrm{~min}$ under darkness. After washing twice with PBS, samples were resuspended in $200 \mu \mathrm{l}$ PBS, then analyzed by flow cytometry (BD 
Biosciences) using FlowJo analysis software (FlowJo, LLC, Ashland, Ore.).

2.8. Real-Time Quantitative Reverse Transcription PCR ( $q R T$ $P C R)$. Total RNA extraction and qRT-PCR were performed as previously described (17).Total RNA was extracted from the lung tissue using TRIzol ${ }^{\circledR}$ reagent (Invitrogen, USA) according to the manufacturer's instructions, then retrotranscribed into cDNA using PrimeScript reverse transcriptase (TaKaRa, Kyoto, Japan) and random primers. qRT-PCR

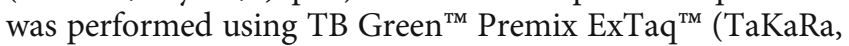
Kyoto, Japan) on a 7500 Real-Time PCR System (Applied Biosystems, USA). The primer sequences used for qRTPCR were MCP-1 forward: $5^{\prime}$-TTA AAA ACC TGG ATC GGA ACC AA-3' and reverse: $5^{\prime}$-GCA TTA GCT TCA GAT TTA CGG GT-3'; CCR-2 forward: $5^{\prime}-\mathrm{ATC}$ CAC GGC ATA CTA TCA ACA TC- $3^{\prime}$ and reverse: $5^{\prime}-\mathrm{CAA}$ GGC TCA CCA TCA TCG TAG-3'; $\beta$-actin forward: $5^{\prime}$ -TGC TGG AAG GTG GAC AGT GAG G-3' and reverse: $5^{\prime}$-CAT TGC TGA CAG GAT GCA GAA GG-3'. The 2 $\triangle \triangle C T$ method was applied to determine relative mRNA expression levels.

2.9. Electrophoretic Mobility Shift Assay (EMSA). EMSA was performed using the RAW 264.7 mouse macrophage cell line. Cells were treated at $43^{\circ} \mathrm{C}$ for $60 \mathrm{~min}$ in a $10 \mathrm{~cm}$ cell culture dish (11). After heating, nuclear extracts were prepared using nuclear and cytoplasmic protein extraction reagents (20126ES50, Yeasen, China). Oligonucleotide probes were synthesized and labeled with biotin by Sangon Biotech, which based on the sequence covering the HSE sequence $(18,19)$ from the promoter regions of $M C P-1$ and CCR2 (Table 1). The following oligonucleotide probes were used (for the sake of clarity, they are shown single-stranded, but double-stranded were used): MCP-1.1: $5^{\prime}$-aggtgagttttatata-

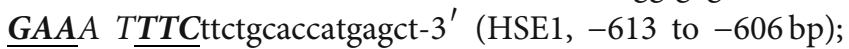
MCP-1.2: 5 '-atctcaggtccagggaagc aTTCTGGAAgcaccagcccca-3' (HSE2, -1475 to $-1468 \mathrm{bp}$ ); CCR2.1: $5^{\prime}$-agcatttaccta GAATTTTCcataacag-3' (HSE1, -754 to $-747 \mathrm{bp}$ ); CCR2.2: $5^{\prime}$-gtttacatTTCTAGAAcc ttatactgtg-3' (HSE2: -1095 to $-1088 \mathrm{bp})$. EMSA was performed using the LightShift ${ }^{\circledR}$ Chemiluminescent EMSA Kit (20148, Thermo Scientific, USA), according to the manufacturer's instructions. The binding reaction was performed as previously described (20). Briefly, biotin-labeled oligonucleotide containing the HSE sequence was incubated with $5 \mu \mathrm{g}$ of nuclear extract for $20 \mathrm{~min}$ at room temperature in binding buffer $(10 \times$ binding buffer, $1 \mu \mathrm{g}$ Poly (dI-dC), 50\% glycerol, 1\% NP-40, $100 \mathrm{mM} \mathrm{MgCl}_{2}, 100 \mathrm{mM}$ EDTA). This reaction was then subjected to gel electrophoresis on a $5 \%$ native polyacrylamide gel and transferred to a nylon membrane. Biotinlabeled DNA was detected by chemiluminescence. For supershift assays, $2 \mu \mathrm{l}$ of anti-HSF1 antibody (ab2923, Abcam, USA) was added to the binding reaction. 200-fold molar excess of the unlabeled competitor oligonucleotides (competitive probe) and the unlabeled mutant oligonucleotides (mutant probe) were used for competition assays.
2.10. Dual-Luciferase Reporter Assay. The constitutively activated HSF1 plasmid pcDNA3.1 (+)/HSF1 (+) (mHSF1) and empty vector plasmid pcDNA3.1 (+)/HSF1-wt were kindly provided by Dr. Richard Voellmy at HSF Pharmaceuticals S.A., Switzerland. Generation of the $M C P-1$ promoter luciferase reporter constructs (the full-length pGL3-MCP-1-wt $(-1600$ to $-1 \mathrm{bp})$ ), pGL3-MCP-1-Mut1 (mutations of HSE1, -613 to $-606 \mathrm{bp}$ ), pGL3-MCP-1-Mut2 (mutations of HSE2, -1475 to -1468 bp), and pGL3-MCP-1-Mut3 (mutations of HSE1 and HSE2)), and the CCR2 promoter luciferase reporter constructs (the full-length pGL3-CCR2-wt (-1162 to $-1 \mathrm{bp}$ ), pGL3-CCR2-Mut1 (mutations of HSE1, -754 to -747 bp), pGL3-MCP-1-Mut2 (mutations of HSE2, -1095 to $-1088 \mathrm{bp}$ ), and pGL3-MCP-1-Mut3 (mutations of HSE1 and HSE2), was performed by PCR and cloned into a luciferase vector pGL3-Basic. The authenticity of the synthesized sequence was verified by sequencing. Plasmids were extracted (D6950-01, Omega, USA) and stored at $-20^{\circ} \mathrm{C}$ for subsequent experiments. RAW 264.7 cells were incubated in a 24 -well plate and grown to $70-90 \%$ confluence, then transfected with $500 \mathrm{ng}$ of the MCP-1 or CCR2 promoter luciferase reporter plasmid, $20 \mathrm{ng}$ of pRL-TK, and $500 \mathrm{ng}$ of mHSF1 plasmid or empty vector plasmid using the Lipofectamine $^{\mathrm{TM}} 3000$ transfection reagent (L3000015, Invitrogen ${ }^{\mathrm{TM}}$, USA). After $48 \mathrm{~h}$ of transfection, the cell lysate was extracted and tested using a dual-luciferase reporter assay kit (E1910, Promega, USA). Luminescence was measured using a Synergy $^{\text {HI }}$ Microplate Reader (BioTek, USA).

2.11. Statistical Analysis. All data were analyzed using GraphPad Prism 7.0 (GraphPad Software, USA) and SPSS 21.0 (SPSS, USA). Data were expressed as mean \pm standard deviation. Student's $t$-test was performed for comparing two groups and one-way analysis of variance (ANOVA) for comparing multiple groups, followed by a multiple comparison test (Bonferroni post hoc test). The Kaplan-Meier analysis was used to compare differences of survival rates between groups. $p<0.05$ was considered statistically significant.

\section{Results}

3.1. HSF1 Alleviated Lung Tissue Damage and Improved Vascular Permeability in LPS-Induced ALI. A dark red congestion was observed on the surface of the lungs from LPSinstillated mice, which was more obvious in the HSF1 /- + LPS group than that in the $\mathrm{HSF}^{+/+}+\mathrm{LPS}$ group (Figure 1(a)). Pathological histomorphology of the lungs revealed increased pulmonary edema, infiltration of inflammatory cells, alveolar hemorrhage, and destruction of the epithelial and endothelial cell structure in the $\mathrm{HSF}^{-/-}+\mathrm{LPS}$ group compared with the symptoms observed in the LPStreated $\mathrm{HSF}^{+/+}$group (Figure $1(\mathrm{~b})$ ). The lung W/D ratio in the $\mathrm{HSF}^{-/-}+$LPS group was significantly higher $(33 \%)$ than that in the $\mathrm{HSF}^{+/+}+\mathrm{LPS}$ group $(p<0.01)$ (Figure $1(\mathrm{c})$ ). The BALF total protein content at 12,24 , and $36 \mathrm{~h}$ after LPS instillation increases significantly in LPS-stimulated mice, showing a significant difference between $\mathrm{HSF} 1^{-/-}+\mathrm{LPS}$ and $\mathrm{HSF}^{+/+}+\mathrm{LPS}$ groups $(p<0.01)$ (Figure $\left.1(\mathrm{~d})\right)$. Moreover, we found that the survival rate of the $\mathrm{HSF} 1^{-/}+\mathrm{LPS}$ group was 
TABLE 1: List of MCP-1 and CCR2 probe sequences.

\begin{tabular}{|c|c|}
\hline Probe & Probe sequence $\left(5^{\prime}-3^{\prime}\right)$ \\
\hline MCP-1.1:labeled probe F: & Biotin-AGGTGAGTTTTATATAGAAATTTCTTCTGCACCATGAGCT \\
\hline MCP-1.1: labeled probe R: & Biotin-AGCTCATGGTGCAGAAGAAATTTCTATATAAAACTCACCT \\
\hline MCP-1.1: competitive probe F: & AGGTGAGTTTTATATAGAAATTTCTTCTGCACCATGAGCT \\
\hline MCP-1.1:competitive probe R: & AGCTCATGGTGCAGAAGAAATTTCTATATAAAACTCACCT \\
\hline MCP-1.1: mutant probe F: & ATGTGAGTGCTATCTAGCACTGTATGCTGCACAATGAGGT \\
\hline MCP-1.1: mutant probe R: & ACCTCATTGTGCAGCATACAGTGCTAGATAGCACTCACAT \\
\hline MCP-1.2: labeled probe F: & Biotin-ATCTCAGGTCCAGGGAAGCATTCTGGAAGCACCAGCCCCA \\
\hline MCP-1.2: labeled probe R: & Biotin-TGGGGCTGGTGCTTCCAGAATGCTTCCCTGGACCTGAGAT \\
\hline MCP-1.2: competitive probe F: & ATCTCAGGTCCAGGGAAGCATTCTGGAAGCACCAGCCCCA \\
\hline MCP-1.2:competitive probe R: & TGGGGCTGGTGCTTCCAGAATGCTTCCCTGGACCTGAGAT \\
\hline MCP-1.2: mutant probe F: & ATATCAGATCCATGGCAGCAGTCTGTACGTACTAGACTCA \\
\hline MCP-1.2: mutant probe R: & TGAGTCTAGTACGTACAGACTGCTGCCATGGATCTGATAT \\
\hline CCR2.1:labeled probe F: & Biotin-AGCATTTACCTAGAATTTTCCATAACAG \\
\hline CCR2.1: labeled probe R: & Biotin-CTGTTATGGAAAATTCTAGGTAAATGCT \\
\hline CCR2.1:competitive probe F: & AGCATTTACCTAGAATTTTCCATAACAG \\
\hline CCR2.1:competitive probe R: & CTGTTATGGAAAATTCTAGGTAAATGCT \\
\hline CCR2.1: mutant probe F: & AGCACTGATCTATGATTAATCATGACAG \\
\hline CCR2.1: mutant probe R: & CTGTCATGATTAATCATAGATCAGTGCT \\
\hline CCR2.2: labeled probe F: & Biotin-GTTTACATTTCTAGAACCTTATACTGTG \\
\hline CCR2.2: labeled probe R: & Biotin-CACAGTATAAGGTTCTAGAAATGTAAAC \\
\hline CCR2.2: competitive probe F: & GTTTACATTTCTAGAACCTTATACTGTG \\
\hline CCR2.2:competitive probe R: & CACAGTATAAGGTTCTAGAAATGTAAAC \\
\hline CCR2.2:mutant probe F: & GTCTATAGGTCTATCACTCTATGCTGTG \\
\hline CCR2.2:mutant probe R: & CACAGCATAGAGTGATAGACCTATAGAC \\
\hline
\end{tabular}

significantly lower than that of the $\mathrm{HSF}^{+/+}+\mathrm{LPS}$ group $(p<0.05)$ (Figure 1(e)). These results showed that lung tissue injury was more severe in the $\mathrm{HSF}^{-/}$group after LPS treatment, indicating that HSF1 alleviated lung tissue damage and improved vascular permeability and survival of LPSinduced ALI mice.

3.2. HSF1 Attenuated Macrophage Infiltration into BALF and Lung Tissue from LPS-Induced ALI Mice. Macrophages are important effector cells in ALI pathogenesis and are closely related to disease severity. To evaluate the macrophage infiltration and exudation in the lung tissue from LPS-treated $\mathrm{HSF}^{+/+}$and $\mathrm{HSF}^{-/-}$mice, we measured the change in percentage of macrophages in BALF at different time points using flow cytometry. The results showed that at $12 \mathrm{~h}, 24 \mathrm{~h}$, and $36 \mathrm{~h}$ after LPS instillation, the macrophage content in BALF from $\mathrm{HSF}^{-/-}$mice was significantly higher than that in $\mathrm{HSF}^{+/+}$mice $(p<0.01)$ (Figures $\left.2(\mathrm{a})-(\mathrm{d})\right)$. Additionally, the tissue immunofluorescence assay results showed that the $\mathrm{HSF}^{-/-}$+ LPS group had a significantly higher macrophage infiltration into the lung tissue than that observed in the $\mathrm{HSF}^{+/+}+\mathrm{LPS}$ group (Figure $2(\mathrm{e})$ ). These results suggested that HSF1 could attenuate macrophage infiltration into the lung tissue from LPS-induced ALI mice.

3.3. HSF1 Reduced the MCP-1 Expression in Serum, Lung Tissue, and BALF from LPS-Induced ALI Mice. Macrophage infiltration is regulated by MCP-1/CCR2 chemokines (2124). MCP-1 levels were measured at different time points in serum, lung tissue, and BALF. At $12 \mathrm{~h}, 24 \mathrm{~h}$, and $36 \mathrm{~h}$ after LPS treatment, the MCP-1 levels in serum from the HSF1 ${ }^{1-}+$ LPS group peaked at $36 \mathrm{~h}$ and were significantly higher than those observed in the HSF $1^{+/+}+\mathrm{LPS}$ group $(p<0.05)$, $(p<0.01)$ (Figure 3(a)). Similar changes were observed in the lung tissue and BALF (Figures 3(b) and 3(c)). Additionally, we found that MCP-1 mRNA levels in the lung tissue were significantly higher in the $\mathrm{HSF}^{-/-}+\mathrm{LPS}$ group than those found in the $\mathrm{HSF} 1^{+/+}+$LPS group $(p<0.05)$ (Figure $3(\mathrm{~d})$ ). Combined with the relative mRNA expression level of HSF1 (Figure 3(e)), these results indicated that HSF1 played a protective role in LPS-induced ALI mice by reducing the MCP-1 expression, thus inhibiting macrophage infiltration.

3.4. HSF1 Reduced the CCR2 Expression in Macrophages from LPS-Induced ALI Mice. Our next question was whether HSF1 affects the expression of CCR2 on the surface of macrophages. The CCR2 expression in mice BALF was detected using flow cytometry. We found that the CCR2 expression increased after $24 \mathrm{~h}$ and $36 \mathrm{~h}$ of LPS treatment, and the difference was more significant after $36 \mathrm{~h} \quad(p<0.01)$ (Figures 4(a)-(d)). Additionally, a tissue immunofluorescence assay was carried out to detect the expression of CCR2 in macrophages from the lung tissue. No significant 


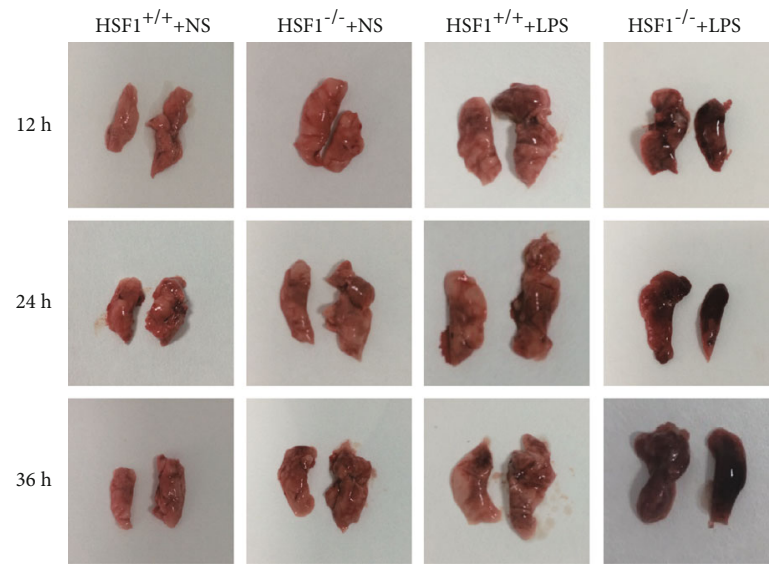

(a)

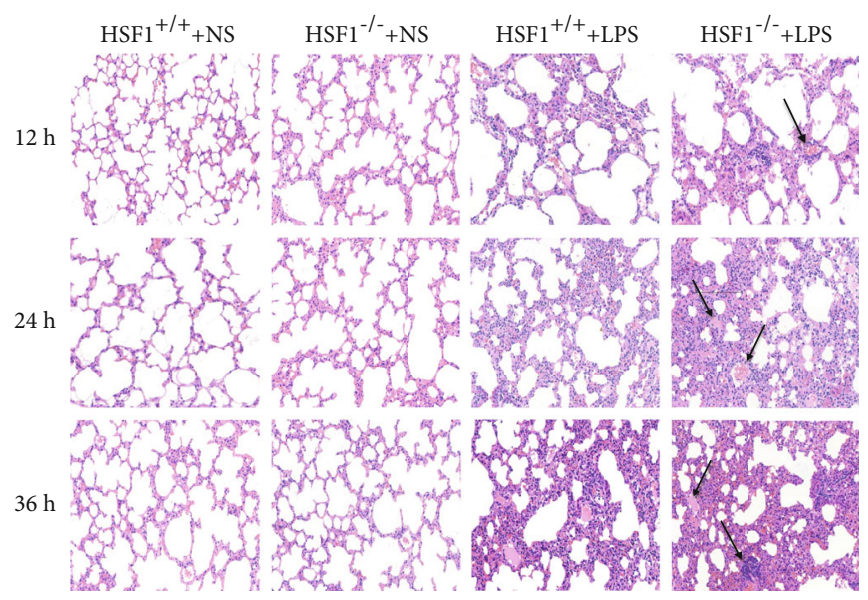

(b)

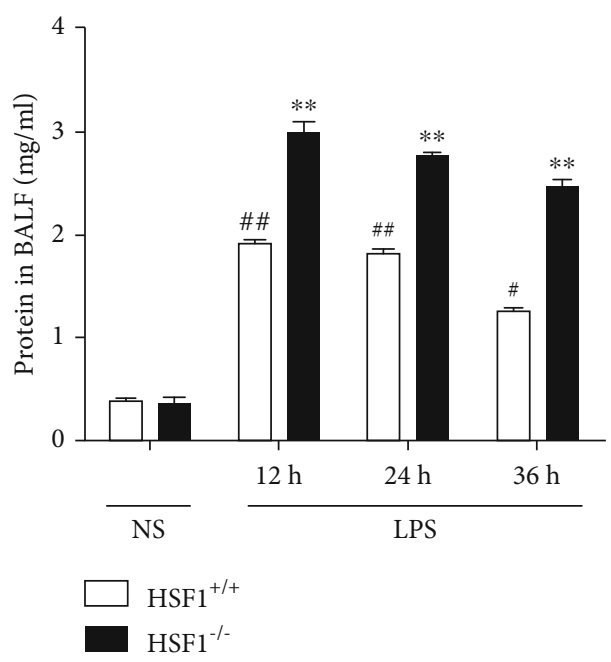

(d)

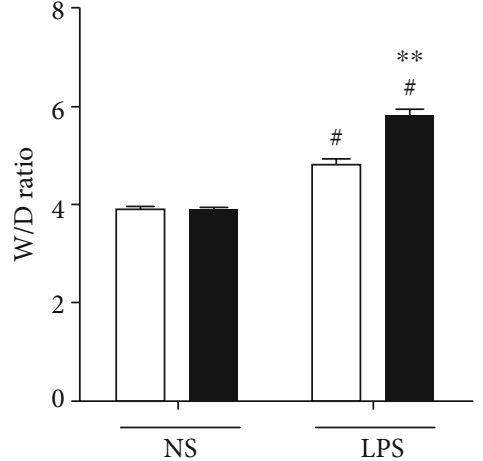

(c)

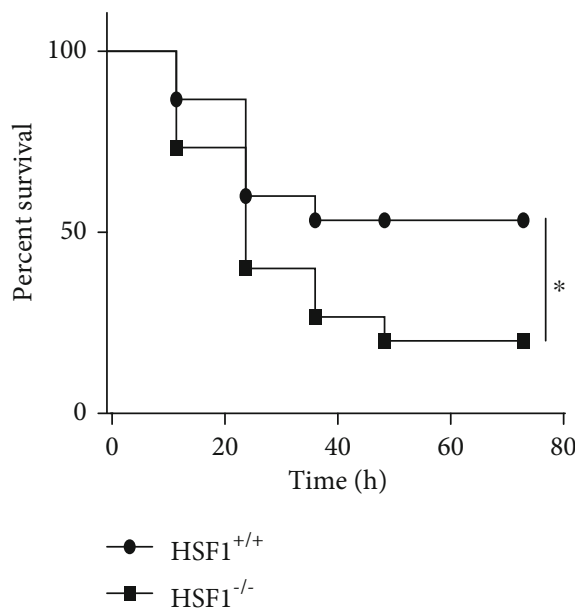

(e)

FIGURE 1: HSF1 reduced lung tissue injury and improved the outcome of LPS-induced ALI mice. (a) Effect of HSF1 on macroscopic changes at $12 \mathrm{~h}, 24 \mathrm{~h}$, and $36 \mathrm{~h}$ after LPS treatment of ALI mice. (b) Representative morphological changes of the lung tissue from LPS-induced ALI mice. Arrows show pulmonary edema, inflammatory cell infiltration, and alveolar hemorrhage (HE staining, $\times 20$ magnification). (c) The lung W/D ratio after $24 \mathrm{~h}$ of LPS treatment of ALI mice. (d) The total protein content in BALF from LPS-induced ALI mice; ${ }^{\#} p<0.05,{ }^{\# \#} p<0.01$, versus $\mathrm{HSF}^{+/+}+\mathrm{NS}$ group; ${ }^{* *} p<0.01$, versus $\mathrm{HSF}^{+/+}+$LPS group; $n=6$ mice per group. (e) The survival rate of LPS-induced ALI mice expressed as a percentage. The survival rate was significantly lower in the HSF $1^{-/-}$group than in wild-type mice $(20.0 \%$ versus $53.3 \%)$ after $72 \mathrm{~h}$ of LPS treatment; $n=15$ mice per group; ${ }^{*} p<0.05$, versus HSF $1^{+/+}$group. $p$ values were determined using two-tailed Student's $t$-test for comparing two groups and one-way ANOVA for comparing multiple groups. 

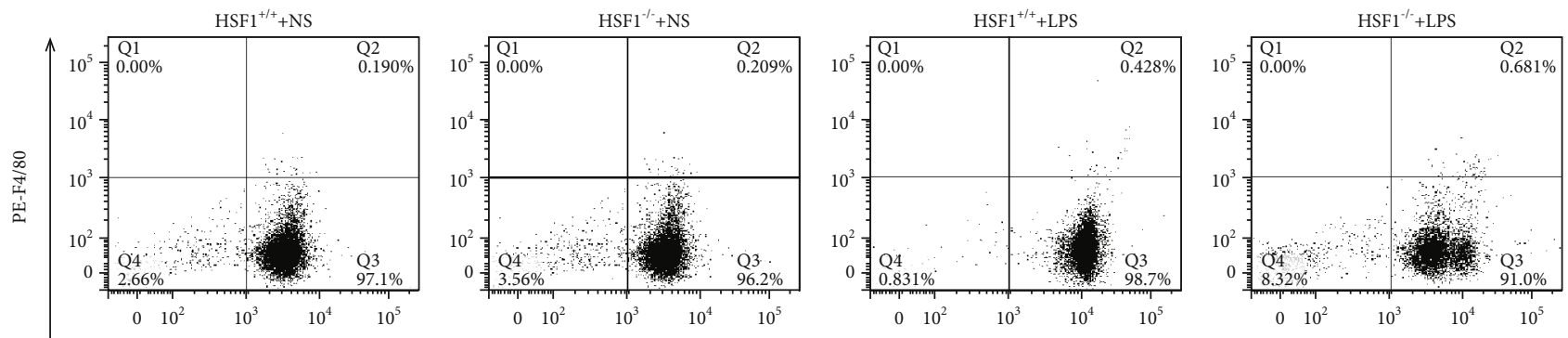

Percp-cy5.5-CD45

(a)
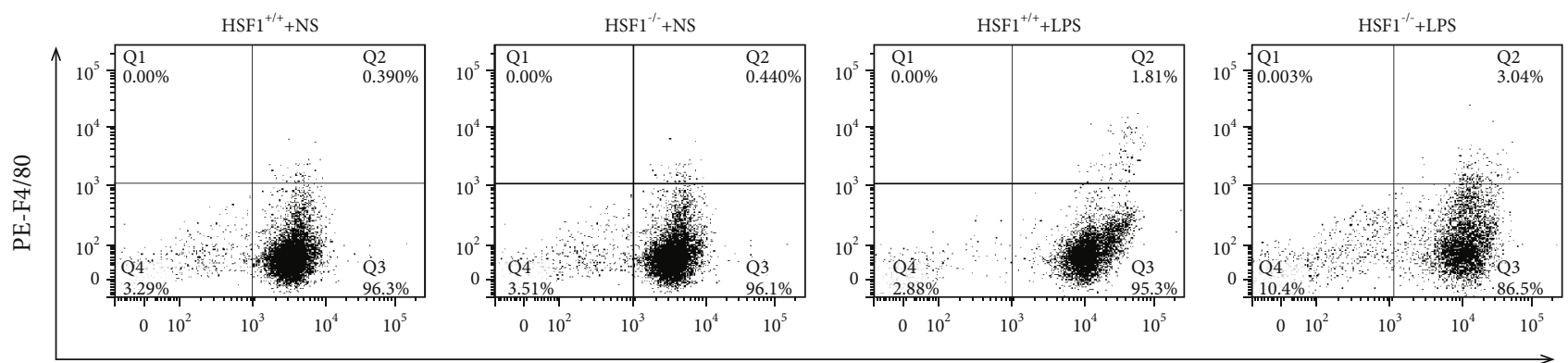

Percp-cy5.5-CD45

(b)
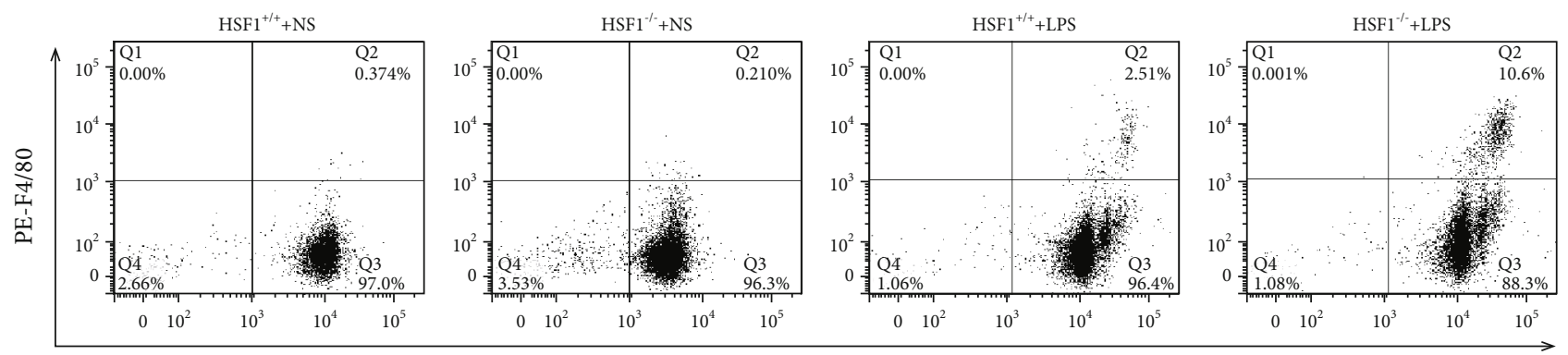

Percp-cy5.5-CD45

(c)
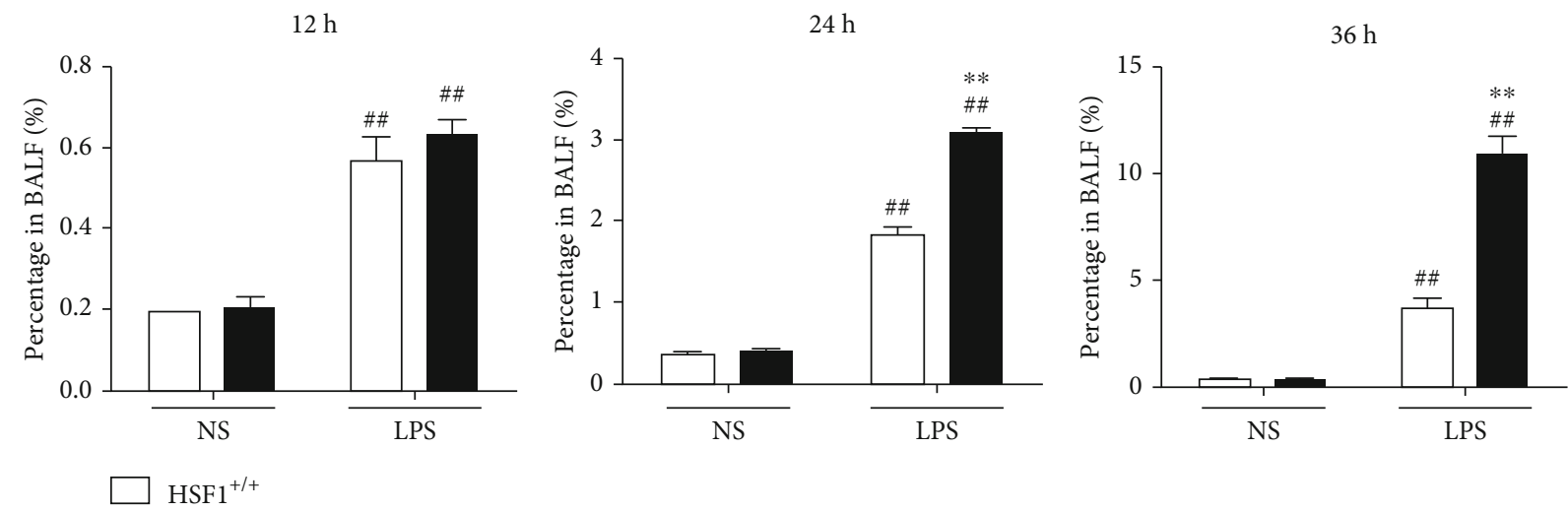

(d)

Figure 2: Continued. 

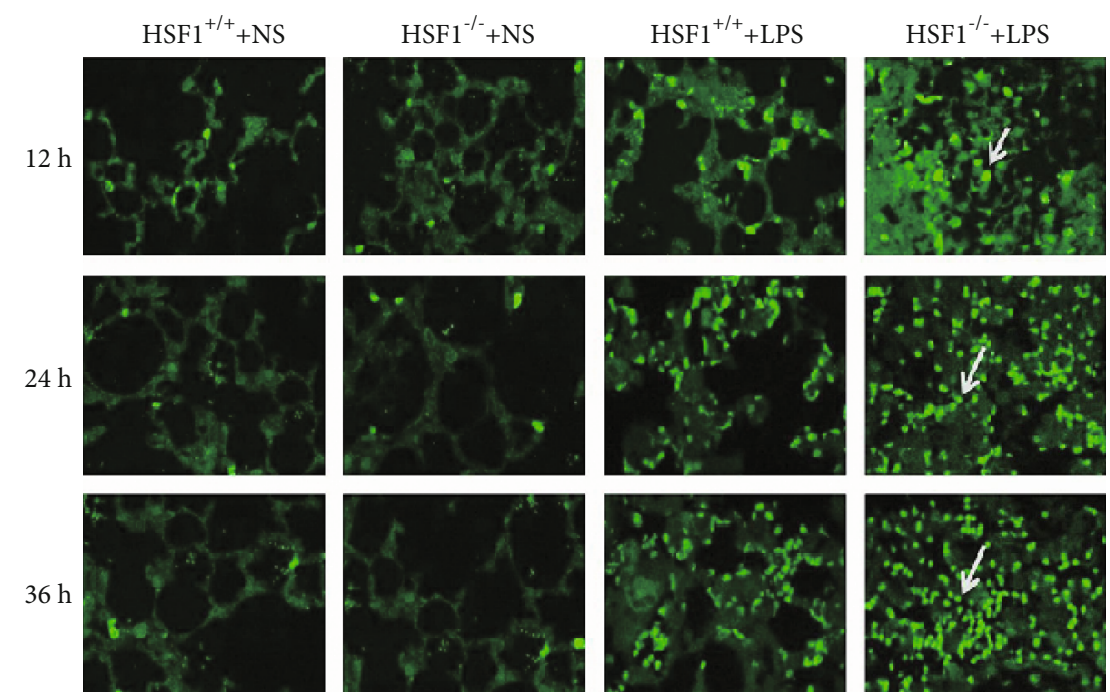

(e)

FIGURE 2: HSF1 reduced macrophage infiltration into the lung tissue from LPS-induced ALI mice. (a)-(d) BALF weas harvested and analyzed for macrophage accumulation using flow cytometry to detect CD $45^{+}$and F4/80 ${ }^{+}$cells at $12 \mathrm{~h}$ (a), $24 \mathrm{~h}$ (b), and $36 \mathrm{~h}$ (c) after LPS treatment. (d) Statistical analysis of (a), (b), (c), respectively. (e) Effect of HSF1 on macrophage infiltration into the lung tissue at $12 \mathrm{~h}, 24 \mathrm{~h}$, and $36 \mathrm{~h}$ after LPS treatment in ALI mice (F4/80, green; white arrowheads indicate individual macrophages; immunofluorescence staining, $\times 40$ magnification); ${ }^{\#} p<0.05,{ }^{\# \#} p<0.01$, versus $\mathrm{HSF}^{+/+}+\mathrm{NS}$ group; ${ }^{*} p<0.05,{ }^{* *} p<0.01$, versus HSF $1^{+/+}+\mathrm{LPS}$ group; $n=6$ mice per group. $p$ values were determined using two-tailed Student's $t$-test for comparing two groups and one-way ANOVA for comparing multiple groups.

difference was found between the expression levels of CCR2 in macrophages from $\mathrm{HSF}^{+/+}$and $\mathrm{HSF}^{-1-}$ mice after $12 \mathrm{~h}$ of LPS treatment (Figure 4(e)). However, at $24 \mathrm{~h}$ and $36 \mathrm{~h}$ after LPS treatment (Figures 4(f) and 4(g)), the CCR2 expression in $\mathrm{HSF}^{-1-}$ mice peaked and was higher than that in $\mathrm{HSF}^{+/+}$mice. Similarly, CCR2 mRNA levels in the lung tissue increased gradually after LPS stimulation, its value being significantly higher in $\mathrm{HSF}^{-1-}$ mice than that in $\mathrm{HSF}^{+/+}$ mice $(p<0.01)$ (Figure $4(\mathrm{~h})$ ). These results indicated that in the LPS-induced ALI model, HSF1 inhibited macrophage infiltration by reducing the expression of CCR2. On the other hand, we proved that by modifying the CCR2 expression, the macrophage infiltration increased significantly in the lung tissue from LPS-treated $\mathrm{HSF}^{-/-}$mice.

3.5. HSF1 Inhibited Monocyte/Macrophage Chemotaxis by Directly Regulating the Transcription of MCP-1/CCR2. In previous studies, we prepared an endotoxemia model using $\mathrm{HSF}^{-1-}$ and $\mathrm{HSF}^{+/+}$mice. We prepared a microarray containing 384 inflammatory factor genes for screening HSF1regulated inflammation-associated genes. It was found that HSF1 inhibited the expression of MCP-1 and its receptor CCR2 (25). A further bioinformatic analysis identified two HSE (HSE1 and HSE2) in the promoters of MCP-1 and CCR2. To conduct the EMSA, we designed probes recognizing the identified HSE sites. Our results showed that HSF1 specifically bound in vitro to HSE1 in both promoters and to HSE2 in the promoter of $M C P-1$ (Figures 5(a), (b), and (d)). To investigate whether HSF1 regulates the MCP-1 and CCR2 expression at the transcriptional level, we performed luciferase assays using reporter constructs containing a DNA fragment with HSE (wt) or with mutant HSE of the MCP-1 and CCR2 promoters, both separately and together
(Figures 5(c) and 5(e)). As shown in Figure 5(c), the HSF1 overexpression resulted in significantly inhibited the promoter activity of MCP-1-wt compared with that observed for the empty vector $(p<0.01)$. The promoter activity of $M C P-1$ was still suppressed by the single HSE1 or HSE2 mutant reporter structure $(p<0.05)$. However, HSF1 did not alter the promoter activity when both two sites were mutated simultaneously. Combined with EMSA results, these findings suggested that the HSF1-binding sites at -613 to $-606 \mathrm{bp}$ (HSE1) and -1475 to $-1468 \mathrm{bp}$ (HSE2) played a major role in regulating the $M C P-1$ promoter activity by HSF1. On the other hand, the luciferase activity of the CCR2-wt reporter was repressed in cells cotransfected with mHSF1 plasmid compared with that observed in the empty vector (Figure 5(e)). However, the repression could be removed when HSE1 was mutated, but not the mutations of HSE2. Combined with EMSA results, these findings suggest that the HSE1 ( -754 to $-747 \mathrm{bp}$ ) played a major role in regulating the CCR2 promoter activity by HSF1. Overall, these results indicated that HSF1 could downregulate the transcription of $M C P-1$ and CCR2, by binding to HSE in their promoter regions.

\section{Discussion}

ALI, or its more severe phenotype acute respiratory distress syndrome (ARDS), can be caused by factors such as severe trauma or infection (26). ARDS affects approximately 200,000 patients every year, resulting in nearly 75,000 deaths in the USA (27). The pathogenesis of ALI is complex and has not been fully elucidated yet. Therefore, further understanding of its pathogenesis has potential therapeutic significance. Based on an ALI model induced by intratracheal instillation 


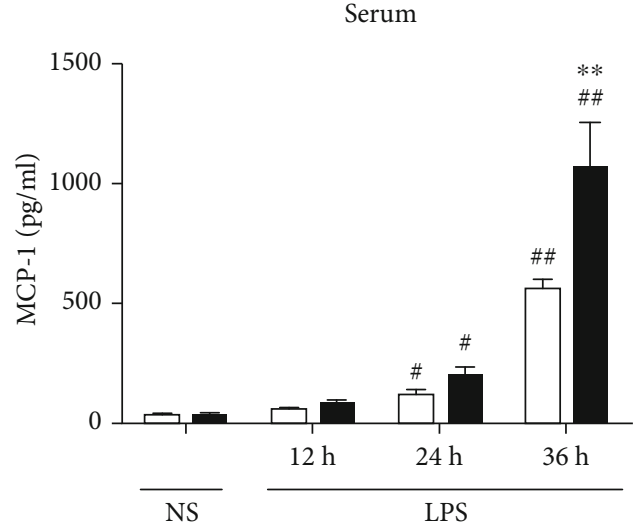

(a)

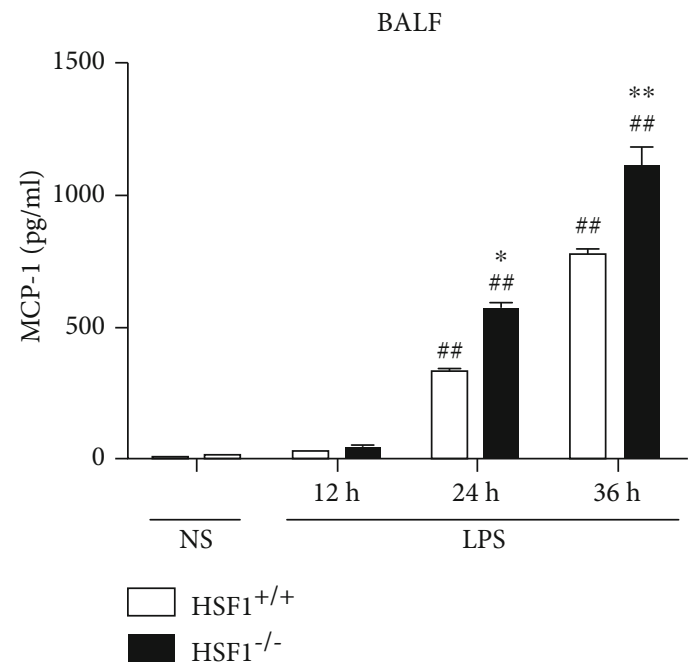

(c)

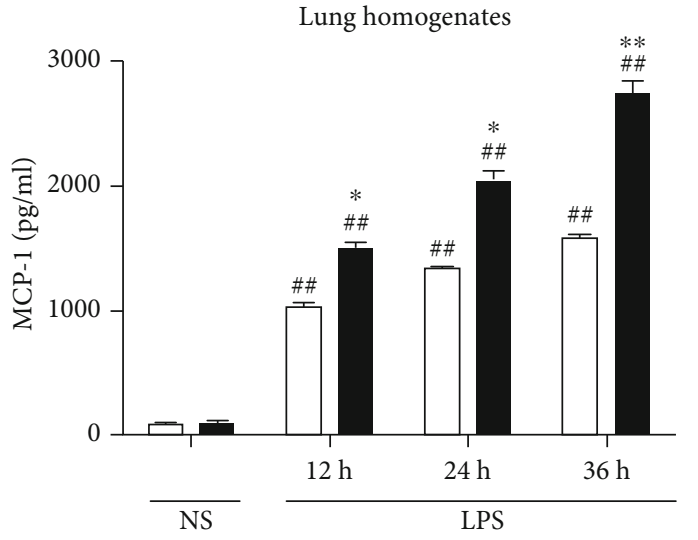

(b)

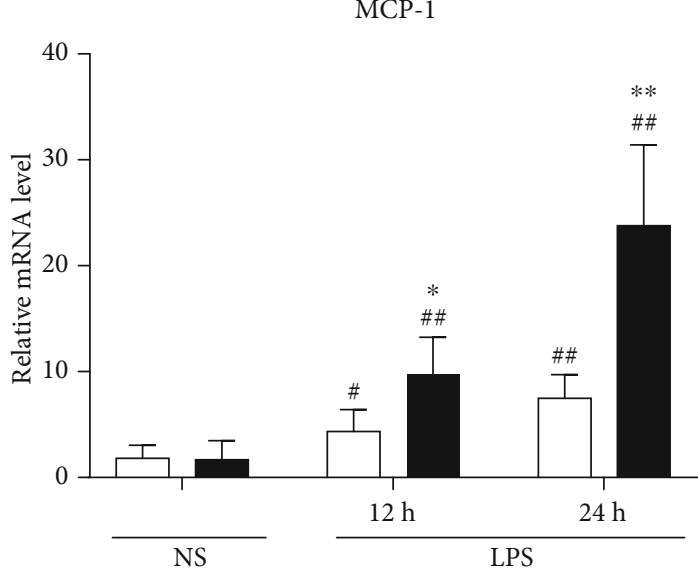

(d)

HSF 1

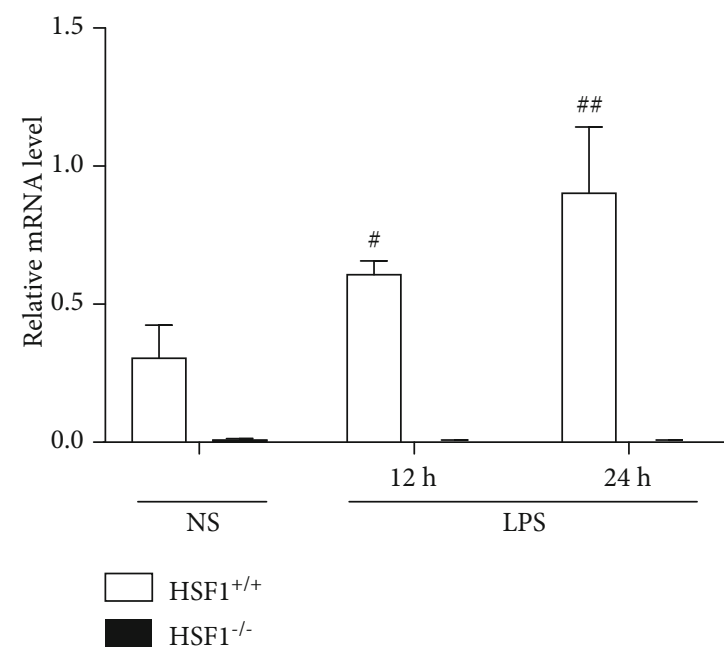

(e)

Figure 3: HSF1 attenuates the MCP-1 expression in serum, lung tissue, and BALF from LPS-induced ALI mice. MCP-1 levels were measured in (a) serum, (b) lung tissue, and (c) BALF at $12 \mathrm{~h}, 24 \mathrm{~h}$, and $36 \mathrm{~h}$ after LPS treatment. (d) MCP-1 mRNA levels in the lung tissue from LPSinduced ALI mice using qRT-PCR. (e) HSF1 mRNA levels in the lung tissue from LPS-induced ALI mice using qRT-PCR. ${ }^{*} p<0.05$, $\# p<0.01$, versus $\mathrm{HSF}^{+/+}+\mathrm{NS}$ group; ${ }^{*} p<0.05,{ }^{* *} p<0.01$, versus HSF $1^{+/+}+$LPS group; $n=6$ mice per group. $p$ values were determined using two-tailed Student's $t$-test for comparing two groups and one-way ANOVA for comparing multiple groups. 

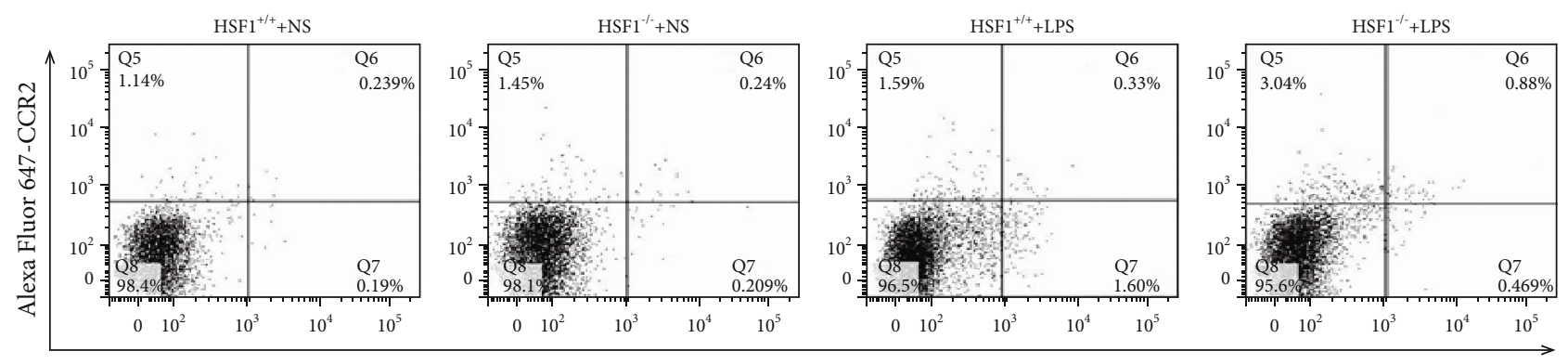

PE-F4/80

(a)
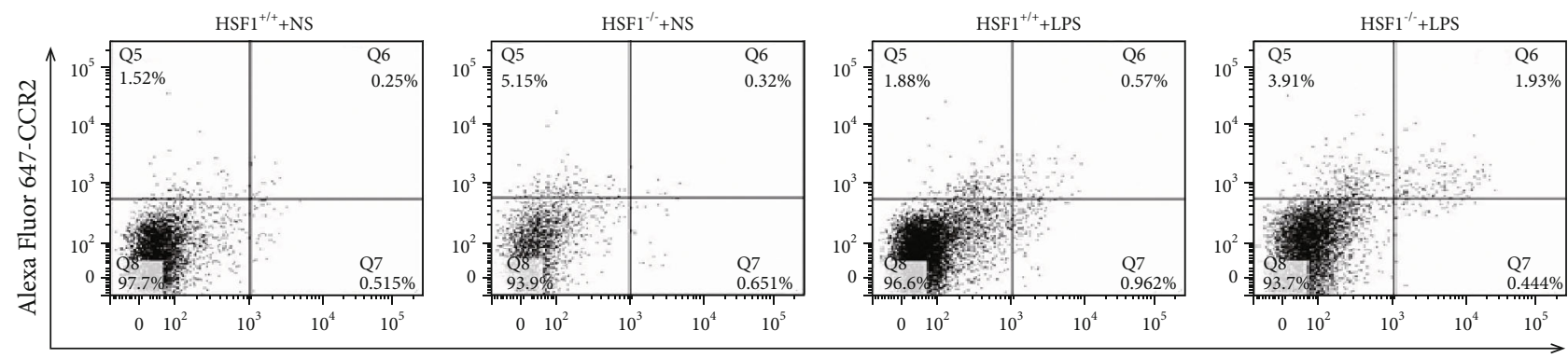

PE-F4/80

(b)
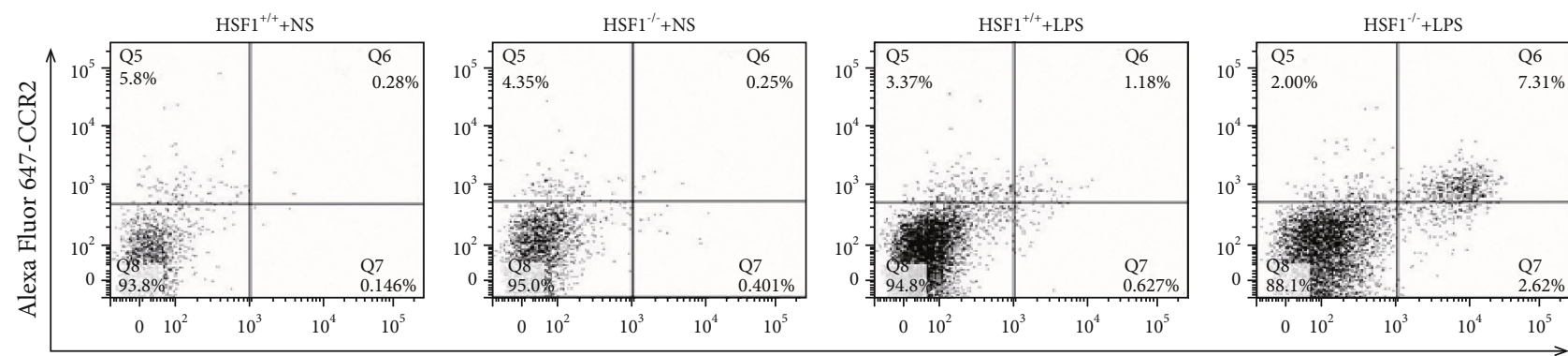

PE-F4/80

(c)

$12 \mathrm{~h}$

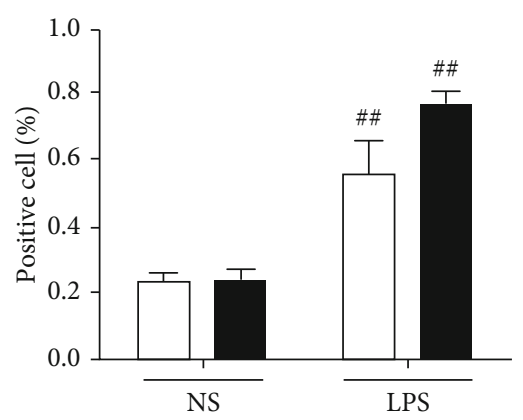

$\square \mathrm{HSFl}^{+/+}$

$\mathrm{HSF}^{-1-}$
$24 \mathrm{~h}$

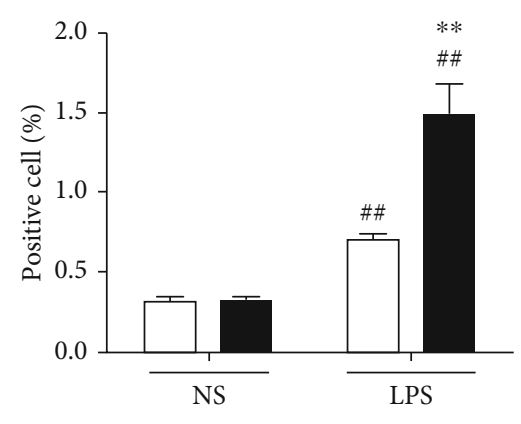

(d)
$36 \mathrm{~h}$

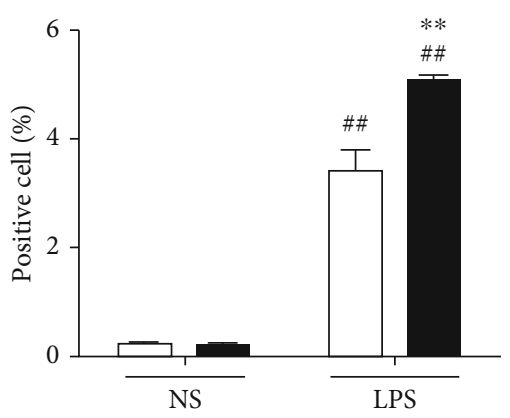

Figure 4: Continued. 

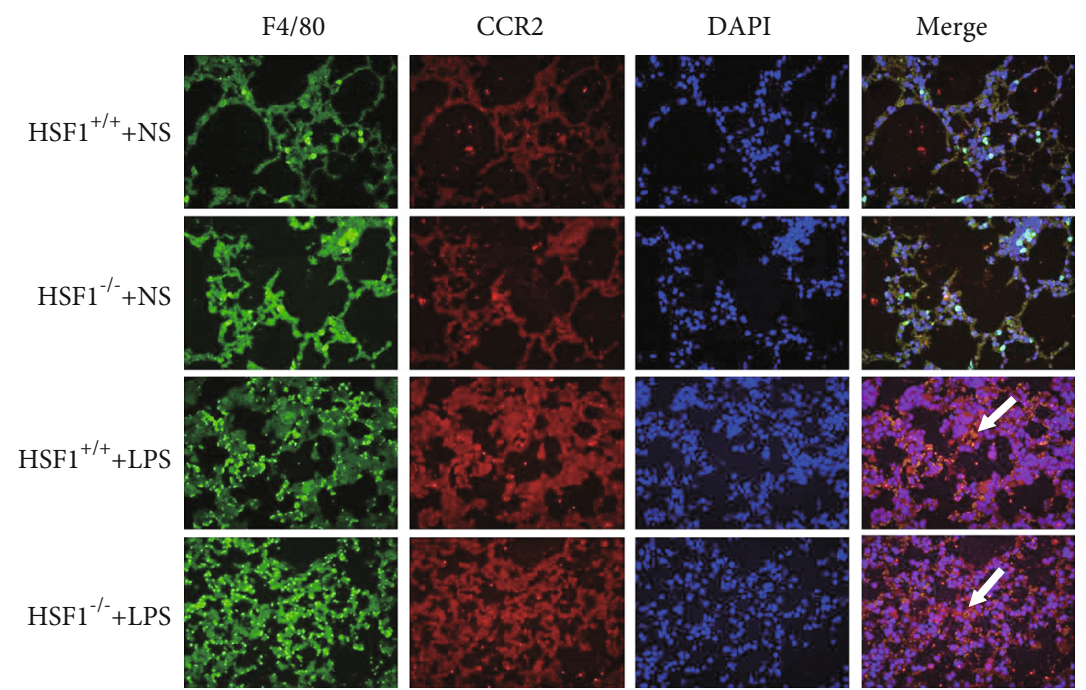

(e)

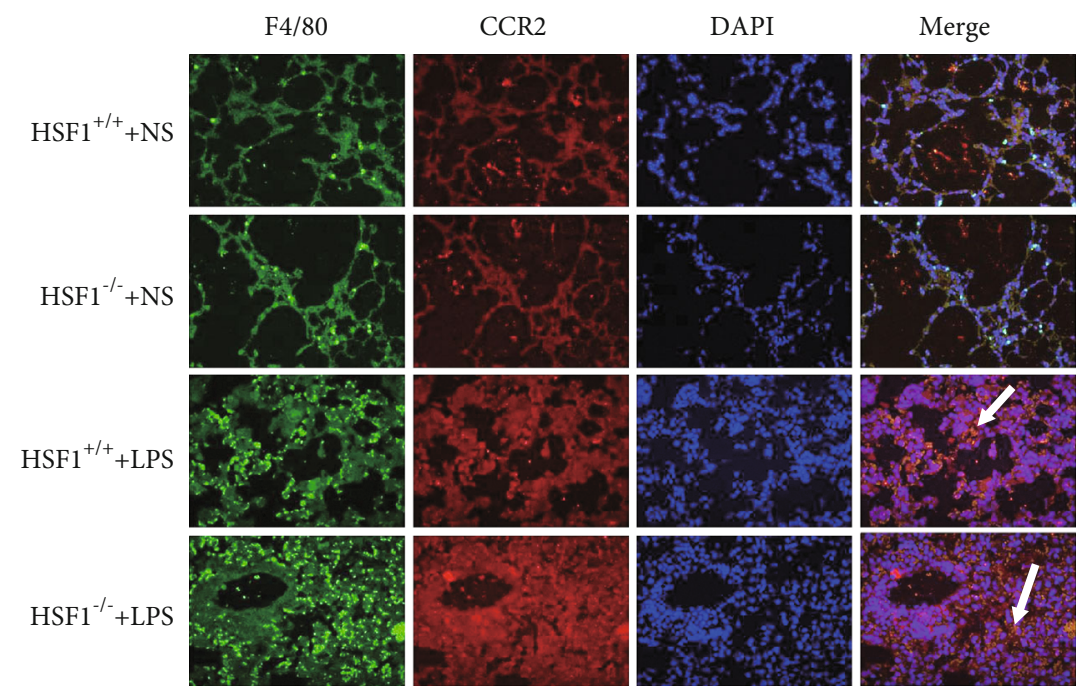

(f)

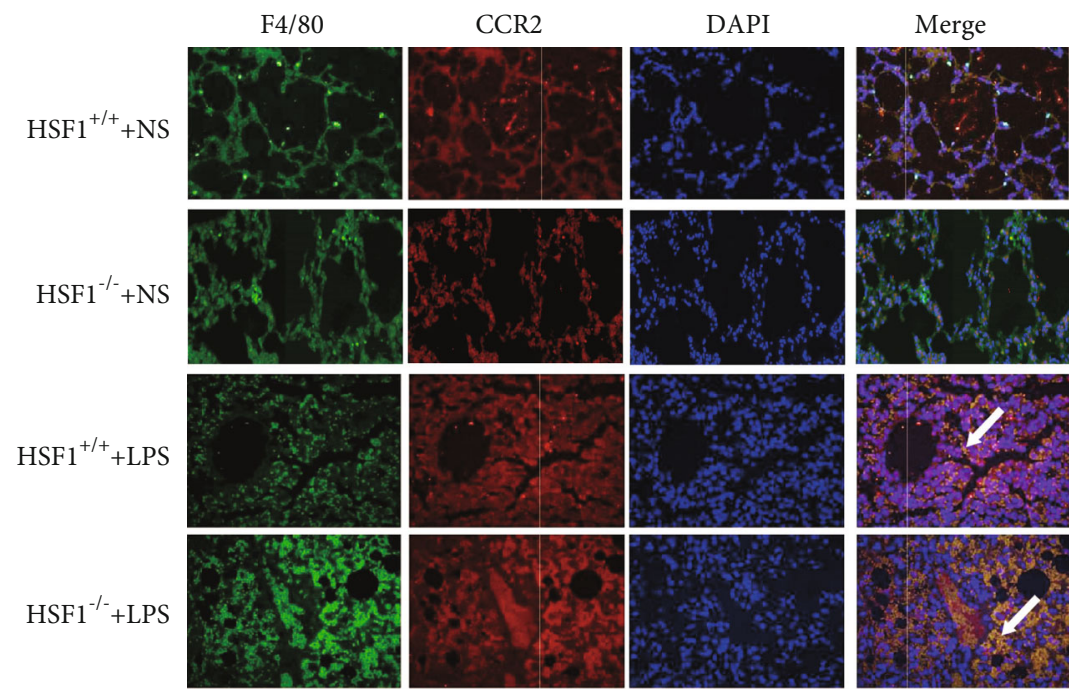

(g)

FIgURe 4: Continued. 


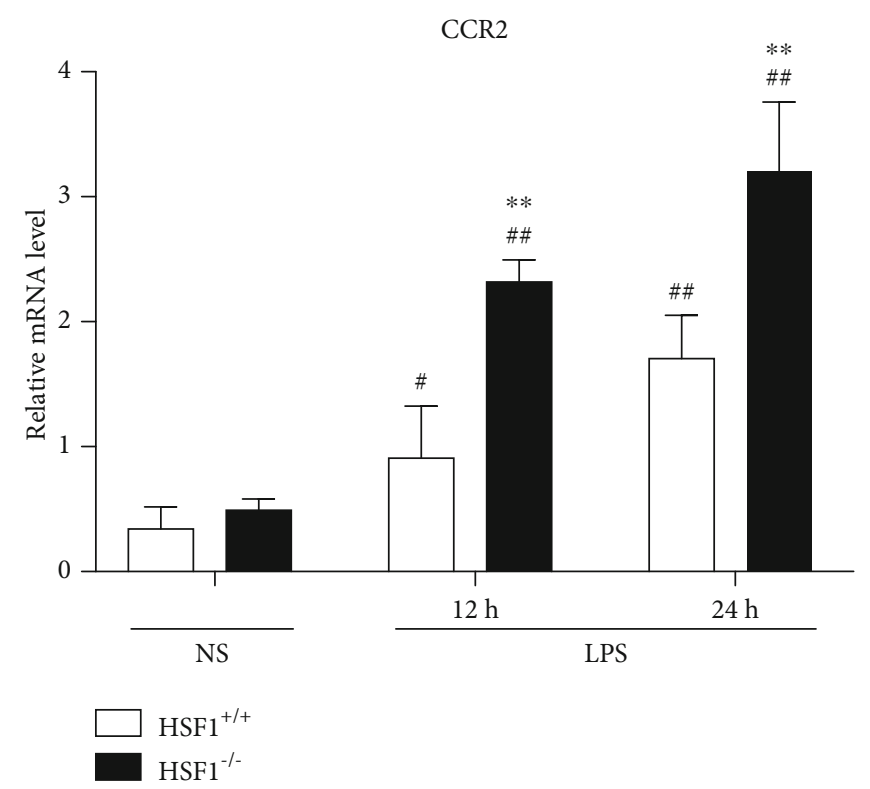

(h)

FIGURE 4: HSF1 reduced the CCR2 expression in macrophages from LPS-induced ALI mice. (a)-(d) BALF samples were analyzed using flow cytometry to measure the CCR2 expression in macrophages $\left(\mathrm{CD} 45^{+}, \mathrm{F} 4 / 80^{+}, \mathrm{CCR} 2^{+}\right.$) at $12 \mathrm{~h}(\mathrm{a}), 24 \mathrm{~h}(\mathrm{~b})$, and $36 \mathrm{~h}$ (c) after LPS treatment. (d) Statistical analysis of (a), (b), and (c). (e)-(g) Immunofluorescence staining of the CCR2 expression (red) in macrophages (green) from the lung tissue at $12 \mathrm{~h}(\mathrm{e}), 24 \mathrm{~h}(\mathrm{f})$, and $36 \mathrm{~h}$ (g) after LPS treatment (F4/80, green; CCR2, red; DAPI, blue; yellow dots indicated by white arrowheads represent the CCR2 expression in macrophages; immunofluorescence staining, $\times 40$ magnification). (h) CCR2 mRNA levels in the lung tissue from LPS-induced ALI mice using qRT-PCR. ${ }^{*} p<0.05,{ }^{\# \#} p<0.01$, versus $\mathrm{HSF}^{+/+}+\mathrm{NS}$ group; ${ }^{*} p<0.05$, ${ }^{* *} p<0.01$, versus $\mathrm{HSF}^{+/+}+\mathrm{LPS}$ group; $n=6$ mice per group. $p$ values were determined using two-tailed Student's $t$-test for comparing two groups and oneway ANOVA for comparing multiple groups.

of LPS in HSF1 knockout mice, this study showed that HSF1 had a protective effect on ALI mice. To the best of our knowledge, we demonstrated for the first time that the protective effect of HSF1 is due to the downregulation of $M C P-1$ and CCR2, owing to the binding of HSF1 to HSE in the promoters of both genes. Consequently, macrophage infiltration was suppressed.

Our previous work showed that HSF1 alleviated multiple organ damages by inhibiting the release of inflammatory factors and leukocyte infiltration into the tissue of endotoxemia mice $(7,8)$. To determine the protective role of HSF1 in LPS-induced ALI, we used HSF1 knockout mice to prepare an ALI model. We found that after LPS stimulation, the lung tissue damage in $\mathrm{HSF}_{-} \mathrm{1}^{-/-}$mice was significantly more severe than that observed in wild-type mice. Furthermore, HSF1 alleviated pulmonary edema, macrophage infiltration, alveolar hemorrhage, reduced protein leakage, and improved the survival of ALI mice. These observations are consistent with those from other studies using LPS-induced ALI models (28, 29). Altogether, these results demonstrated that HSF1 could alleviate lung damage, improved the survival rate, and had a protective effect against LPS-induced ALI.

The inflammatory response is a crucial element in the pathogeneses of ALI; its pathophysiological features are inflammatory exudation and an imbalance between proinflammatory and anti-inflammatory responses, which may eventually lead to respiratory failure (30). The evidence suggests that macrophages are key cells in the pathogenesis of
ALI/ARDS (31, 32). Macrophage recruitment into the damaged tissue is an important process leading to inflammatory damage. Some studies show that MCP-1 and its receptor CCR2 play important roles in macrophage migration (2124). MCP-1 (also known as CCL2) is a member of the C-C chemokine family and binds to CCR2 (33). MCP-1 is usually secreted by macrophages in response to pathogen infection (34) and is the most potent inducer of the signal transduction pathways leading to monocyte transmigration (35). The high affinity of MCP-1 for CCR2 is an important factor in promoting monocyte/macrophage activation, chemotaxis, and inflammatory responses, resulting in important biological consequences $(21,22)$. To confirm the effect of HSF1 on macrophage infiltration, we analyzed serum, lung tissue homogenates, and BALF from ALI mice to quantify the concentration of MCP-1. Further, we analyzed the expression of CCR2 on the surface of macrophages in BALF and lung tissue using flow cytometry and immunofluorescence assay, respectively. The results showed that the expression of MCP-1 in serum, lung tissue, and BALF from LPSstimulated $\mathrm{HSF}^{-/-}$mice gradually increased after stimulation and was significantly higher than that observed in wild-type mice. The expression of CCR2 on the surface of macrophages was concomitant with the MCP-1 expression.

The results suggested that HSF1 inhibited macrophage migration by downregulating both MCP-1 and CCR2. However, the downregulating mechanism is unclear. It has been proved that HSF1 plays a role in several diseases by regulating gene transcription (36-38). The binding of HSF1 to a 


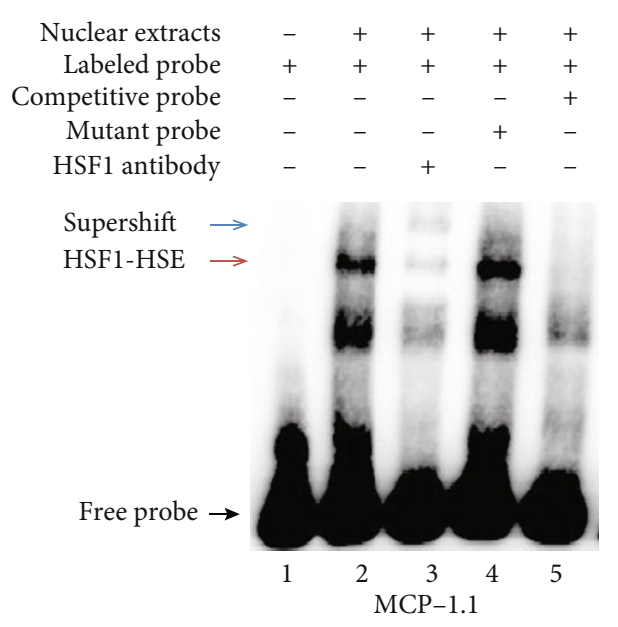

(a)

$\begin{array}{rccccc}\text { Nuclear extracts } & - & + & + & + & + \\ \text { Labeled probe } & + & + & + & + & + \\ \text { Competitive probe } & - & - & - & - & + \\ \text { Mutant probe } & - & - & - & + & - \\ \text { HSF1 antibody } & - & - & + & - & -\end{array}$

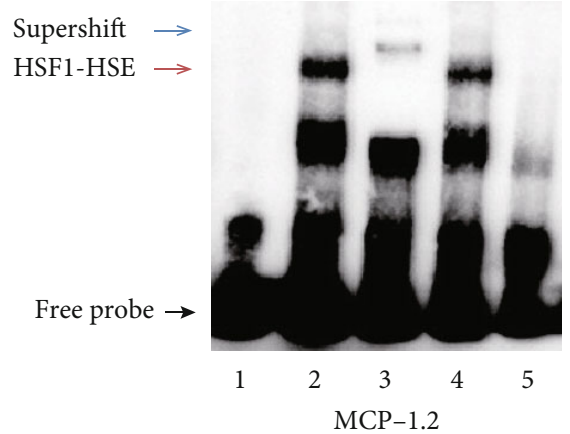

(b)

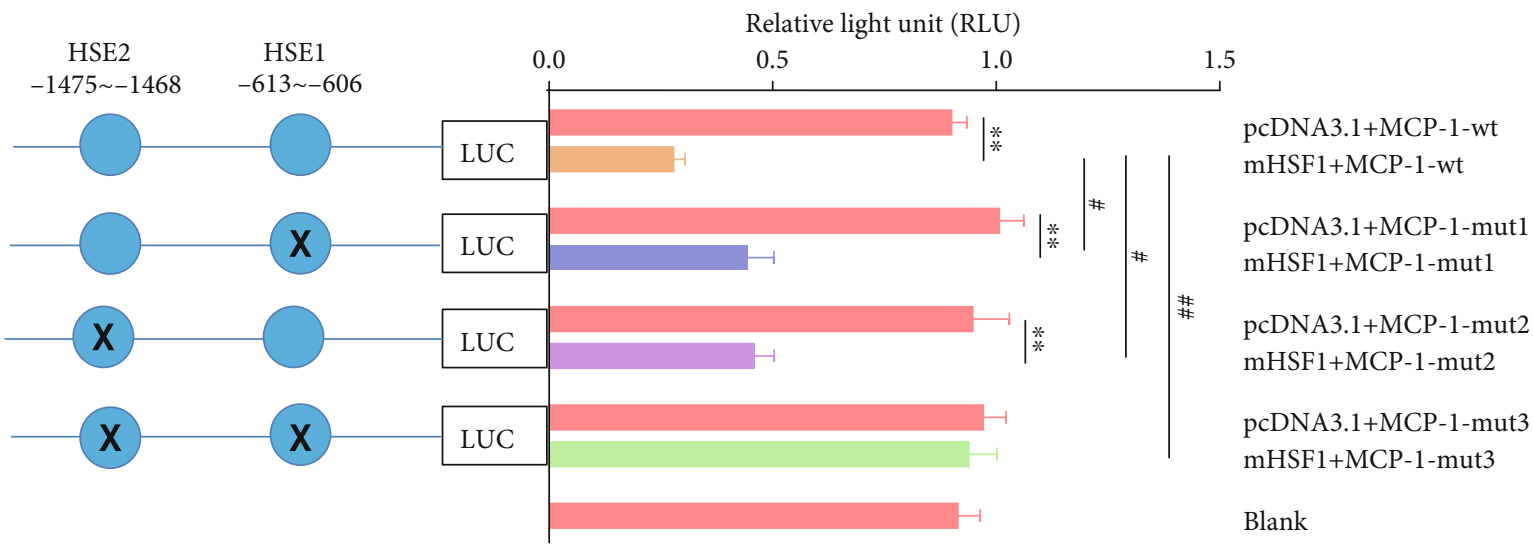

MCP-1

(c)

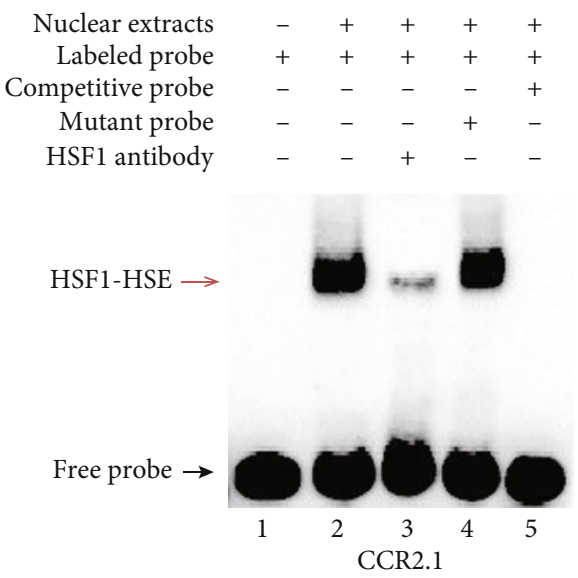

(d)

Figure 5: Continued. 


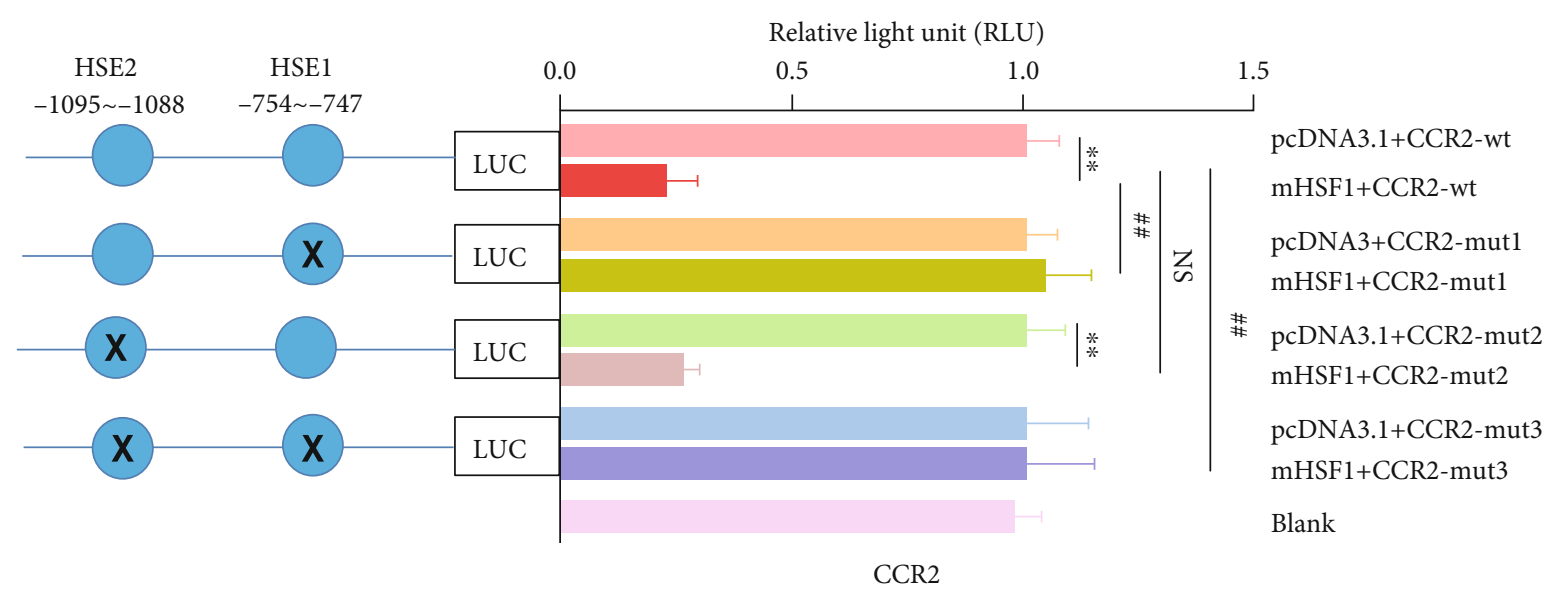

(e)

FIGURE 5: HSF1 downregulated the transcription of MCP-1/CCR2. (a, b, d) The binding of HSF1 to HSE in MCP-1 and CCR2 promoters was assessed using EMSA. $(\mathrm{a}, \mathrm{b})$ EMSA was used to detect the binding of the HSF1 protein with HSE1 (a) and HSE2 (b) in the MCP-1 promoter region in vitro. The biotin probe specific for HSE1 or HSE2 was bound by nuclear extracts of RAW 264.7 cells, which could be blocked by an HSF1 antibody. (d) EMSA was used to detect the binding of the HSF1 protein with HSE1 in the CCR2 promoter region in vitro. The biotin probe specific for HSE1 was bound by nuclear extracts, which could be blocked by an HSF1 antibody. Lane 1: negative control; lane 2: biotinlabeled HSE probe preincubated with nucleoproteins (HSF1-HSE complexes); lane 3: supershift analysis using anti-HSF1 antibodies; lane 4: competition using a 200-fold excess of the unlabeled competitive probe; lane 5: competition using a 200-fold excess of the unlabeled mutant probe. The HSF1/HSE complex is indicated by red arrowheads, the supershift band is indicated by blue arrowheads, and unbound HSE probe is indicated by black arrowheads. (c, e) Luciferase reporter assay analysis of HSF1 regulation on the promoter transcription activity of $M C P-1$ (c) and CCR2 (e) in RAW 264.7 cells transfected with the HSF1 overexpression vector (mHSF1) or empty expression vector pcDNA3.1. After $48 \mathrm{~h}$ of transfection, the cell lysate was extracted, and the firefly luciferase activity was measured and normalized to the Renilla luciferase activity. ${ }^{* *} p<0.01$, pcDNA3.1 versus mHSF1 (intragroup comparison); ${ }^{*} p<0.05$, versus mHSF1 + MCP-1-wt (or mHSF1 + CCR2-wt); $\# p<0.01$, versus mHSF1 + MCP-1-wt (or mHSF1 + CCR2-wt). Data are representative of at least three independent experiments. $p$ values were determined using two-tailed Student's $t$-test for comparing two groups and one-way ANOVA for comparing multiple groups.

gene promoter is a key step to activate gene transcription. Under stress conditions, HSF1 forms homotrimers that upon phosphorylation bind to the promoter of heat shock genes to regulate their transcription (39-41). HSF1 can regulate the expression of other genes, including inflammatory cytokines $(37,42,43)$. To explore the effect of HSF1 on inflammation, in previous studies, we screened for inflammationassociated genes potentially regulated by HSF1, using a microarray containing inflammatory cytokine genes. We found that HSF1 regulated the expression of several genes including $M C P-1 / C C R 2$ (25). Further, bioinformatic analysis showed that there were several HSE in the promoters of $M C P-1 / C C R 2$. Combined with the results from the JASPAR core database, we proposed a hypothesis on how HSF1 may affect the course of inflammation by directly regulating the expression of $M C P-1 / C C R 2$. In this study, we tested such hypothesis by using EMSA and dual luciferase reporter assay experiments and found that HSF1 could directly downregulate MCP-1/CCR2. Therefore, the protective effect of HSF1 on ALI mice would be explained by the inhibition of macrophage migration and infiltration, owing to the downregulation of $M C P-1 / C C R 2$.

\section{Conclusion}

In summary, HSF1 attenuated LPS-induced ALI in mice by suppressing macrophage infiltration owing to the downregulation of $M C P-1 / C C R 2$.

\section{Abbreviations}

ALI: $\quad$ Acute lung injury

ARDS: $\quad$ Acute respiratory distress syndrome

BALF: Bronchoalveolar lavage fluids

BSA: Bovine serum albumin

CCR2: $\quad$ Chemokine (C-C motif) receptor 2

ELISA: Enzyme-linked immunosorbent assay

EMSA: Electrophoretic mobility shift assay

HSE: Heat shock element

HSF1: $\quad$ Heat shock factor 1

LPS: Lipopolysaccharide

MODS: Multiorgan dysfunction syndrome

MCP-1: Monocyte chemoattractant protein-1

NS: $\quad$ Normal saline

PBS: $\quad$ Phosphate-buffered saline

qRT-PCR: Real-time quantitative reverse transcription PCR W/D: Wet/dry weight.

\section{Data Availability}

The data used to support the findings of this study are available from the corresponding author upon request.

\section{Conflicts of Interest}

The authors declare no conflict of interest, and the work described here has not been published previously and not 
under consideration for publication elsewhere, in whole or in part.

\section{Acknowledgments}

This study is supported by the Hainan Provincial Natural Science Foundation of China, Grant No. 819QN229; Training Program of the Hainan Medical University, Grant No. HY2018-33; the National Natural Science Fund of China, Grant No. 81671895, 81871610, 81471897, and 81870071; Natural Science Foundation of Hunan Province, China, Grant No. 2019JJ40393.

\section{References}

[1] P. Arthur and G. R. B. Wheeler, "Acute lung injury and the acute respiratory distress syndrome: a clinical review," The Lancet, vol. 369, no. 9572, pp. 1553-1564, 2007.

[2] L. B. Ware, "Pathophysiology of acute lung injury and the acute respiratory distress syndrome," Seminars in Respiratory and Critical Care Medicine, vol. 27, no. 4, pp. 337349, 2006.

[3] J. Villar, J. D. Edelson, M. Post, J. B. Mullen, and A. S. Slutsky, "Induction of heat stress proteins is associated with decreased mortality in an animal model of acute lung injury," The American Review of Respiratory Disease, vol. 147, no. 1, pp. 177-181, 1993.

[4] J. Villar, S. P. Ribeiro, J. B. Mullen, M. Kuliszewski, M. Post, and A. S. Slutsky, "Induction of the heat shock response reduces mortality rate and organ damage in a sepsis-induced acute lung injury model," Critical Care Medicine, vol. 22, no. 6, pp. 914-921, 1994.

[5] M. Pespeni, R. C. Mackersie, H. Lee et al., "Serum levels of Hsp60 correlate with the development of acute lung injury after Trauma1," The Journal of Surgical Research, vol. 126, no. 1, pp. 41-47, 2005.

[6] D. S. Wheeler and H. R. Wong, "The heat shock response and acute lung injury," Free Radical Biology and Medicine, vol. 42, no. 1, pp. 1-14, 2007.

[7] S. Chen, X. Zuo, M. Yang et al., "Severe multiple organ injury in HSF1 knockout mice induced by lipopolysaccharide is associated with an increase in neutrophil infiltration and surface expression of adhesion molecules," Journal of Leukocyte Biology, vol. 92, no. 4, pp. 851-857, 2012.

[8] B. J. Zhongyi Tong and L. Zhang, "HSF-1 is involved in attenuating the release of inflammatory cytokines induced by LPS through regulating autophagy," SHOCK, vol. 41, no. 5, p. 449Y453, 2014.

[9] A. E. Williams and R. C. Chambers, "The mercurial nature of neutrophils: still an enigma in ARDS?," American Journal of Physiology-Lung Cellular and Molecular Physiology, vol. 306, no. 3, pp. L217-L230, 2014.

[10] X. Xiao, "HSF1 is required for extra-embryonic development, postnatal growth and protection during inflammatory responses in mice," The EMBO Journal, vol. 18, no. 21, pp. 5943-5952, 1999.

[11] D. R. McMillan, X. Xiao, L. Shao, K. Graves, and I. J. Benjamin, "Targeted disruption of heat shock transcription factor 1 abolishes thermotolerance and protection against heat-inducible apoptosis," The Journal of Biological Chemistry, vol. 273, no. 13 , pp. $7523-7528,1998$.
[12] L. Jiang, L. Zhang, K. Kang et al., "Resveratrol ameliorates LPSinduced acute lung injury via NLRP3 inflammasome modulation," Biomedicine \& pharmacotherapy, vol. 84, pp. 130-138, 2016.

[13] A. M. Wolfe, L. H. Kennedy, J. J. Na, and J. A. Nemzek-Hamlin, "Efficacy of tramadol as a sole analgesic for postoperative pain in male and female mice," Journal of the American Association for Laboratory Animal Science, vol. 54, no. 4, pp. 411419, 2015.

[14] G. Dos Santos, M. R. Rogel, M. A. Baker et al., "Vimentin regulates activation of the NLRP3 inflammasome," Nature communications, vol. 12, no. 1, pp. 1-13, 2015.

[15] L. S. de Aragão-França, L. S. Aragão-França, V. C. J. Rocha et al., "Tolerogenic dendritic cells reduce airway inflammation in a model of dust mite triggered allergic inflammation," Allergy, Asthma \& Immunology Research, vol. 10, no. 4, pp. 406-419, 2018.

[16] A. Pierre, F. Lemaire, A. Meghraoui-Kheddar, S. Audonnet, S. Héry-Huynh, and R. Le Naour, "Impact of aging on inflammatory and immune responses during elastin peptide-induced murine emphysema," American Journal of Physiology. Lung Cellular and Molecular Physiology, vol. 316, no. 4, pp. L608L620, 2019.

[17] J. Hu, W. Zhang, Y. Liu et al., "LDK378 inhibits the recruitment of myeloid-derived suppressor cells to spleen via the p38-GRK2-CCR2 pathway in mice with sepsis," Immunology and Cell Biology, vol. 97, no. 10, pp. 902-915, 2019.

[18] C. J. Goldenberg, Y. Luo, M. Fenna, R. Baler, R. Weinmann, and R. Voellmy, "Purified human factor activates heat shock promoter in a HeLa cell-free transcription system," The Journal of Biological Chemistry, vol. 263, no. 36, pp. 1973419739, 1988.

[19] H. S. Choi, Z. Lin, B. S. Li, and A. Y. Liu, "Age-dependent decrease in the heat-inducible DNA sequence-specific binding activity in human diploid fibroblasts," The Journal of Biological Chemistry, vol. 265, no. 29, pp. 18005-18011, 1990.

[20] L. Zhang, M. Yang, Q. Wang et al., "HSF1 regulates expression of G-CSF through the binding element for NF-IL6/CCAAT enhancer binding protein beta," Molecular and Cellular Biochemistry, vol. 352, no. 1-2, pp. 11-17, 2011.

[21] S. Bose and J. Cho, "Role of chemokine CCL2 and its receptor CCR2 in neurodegenerative diseases," Archives of Pharmacal Research, vol. 36, no. 9, pp. 1039-1050, 2013.

[22] J. C. Lima-Junior, R. N. Rodrigues-da-Silva, V. A. Pereira et al., "Cells and mediators of inflammation (C-reactive protein, nitric oxide, platelets and neutrophils) in the acute and convalescent phases of uncomplicated plasmodium vivax and plasmodium falciparum infection," Memórias do Instituto Oswaldo Cruz, vol. 107, no. 8, pp. 1035-1041, 2012.

[23] T. Okuma, Y. Terasaki, N. Sakashita et al., "MCP-1/CCR2 signalling pathway regulates hyperoxia-induced acute lung injury via nitric oxide production," International Journal of Experimental Pathology, vol. 87, no. 6, pp. 475-483, 2006.

[24] B. Z. Qian, J. Li, H. Zhang et al., "CCL2 recruits inflammatory monocytes to facilitate breast-tumour metastasis," Nature, vol. 475, no. 7355, pp. 222-225, 2011.

[25] F. X. Yu, H. L. Zhang, G. W. Chen et al., "Screen of inflammatory genes regulated by heat shock factor 1 and corroboration w ith SOCS3 gene," Journal of Central South University. Medical sciences, vol. 31, no. 2, pp. 167-173, 2006. 
[26] A. S. Slutsky, J. Villar, and A. Pesenti, "Happy 50th birthday ARDS!," Intensive Care Medicine, vol. 42, no. 5, pp. 637-639, 2016.

[27] G. D. Rubenfeld, E. Caldwell, E. Peabody et al., "Incidence and outcomes of acute lung injury," The New England Journal of Medicine, vol. 353, no. 16, pp. 1685-1693, 2005.

[28] A. Pfalzgraff, L. Heinbockel, Q. Su, T. Gutsmann, K. Brandenburg, and G. Weindl, "Synthetic antimicrobial and LPS-neutralising peptides suppress inflammatory and immune responses in skin cells and promote keratinocyte migration," Scientific Reports, vol. 6, no. 31577, pp. 1-12, 2016.

[29] Z. Zhang, J. Zhou, D. Song, Y. Sun, C. Liao, and X. Jiang, "Gastrodin protects against LPS-induced acute lung injury by activating Nrf2 signaling pathway," Oncotarget, vol. 8, no. 19, pp. 32147-32156, 2017.

[30] F. Gerin, U. Sener, H. Erman et al., "The effects of quercetin on acute lung injury and biomarkers of inflammation and oxidative stress in the rat model of sepsis," Inflammation, vol. 39, no. 2, pp. 700-705, 2016.

[31] J. Lomas-Neira, C. S. Chung, M. Perl, S. Gregory, W. Biffl, and A. Ayala, "Role of alveolar macrophage and migrating neutrophils in hemorrhage-induced priming for ALI subsequent to septic challenge," American Journal of Physiology-Lung Cellular and Molecular Physiology, vol. 290, no. 1, pp. L51-L58, 2006.

[32] L. K. Johnston, C. R. Rims, S. E. Gill, J. K. McGuire, and A. M. Manicone, "Pulmonary macrophage subpopulations in the induction and resolution of acute lung injury," American Journal of Respiratory Cell and Molecular Biology, vol. 47, no. 4, pp. 417-426, 2012.

[33] P. E. Kolattukudy and J. Niu, "Inflammation, endoplasmic reticulum stress, autophagy, and the monocyte chemoattractant protein-1/CCR2 pathway," Circulation Research, vol. 110, no. 1, pp. 174-189, 2012.

[34] G. H. Tesch, "MCP-1/CCL2: a new diagnostic marker and therapeutic target for progressive renal injury in diabetic nephropathy," American Journal of Physiology. Renal Physiology, vol. 294, no. 4, pp. F697-F701, 2008.

[35] S. Sozzani, D. Zhou, M. Locati et al., "Receptors and transduction pathways for monocyte chemotactic protein-2 and monocyte chemotactic protein-3. Similarities and differences with MCP-1," Journal of Immunology, vol. 152, no. 7, pp. 36153622, 1994.

[36] C. M. Cahill, W. R. Waterman, Y. Xie, P. E. Auron, and S. K. Calderwood, "Transcriptional repression of the prointerleukin lbeta gene by heat shock factor 1," The Journal of Biological Chemistry, vol. 271, no. 40, pp. 24874-24879, 1996.

[37] Y. Xie, C. Chen, M. A. Stevenson, P. E. Auron, and S. K. Calderwood, "Heat shock factor 1 represses transcription of theIL- $1 \beta$ Gene through physical interaction with the nuclear factor of interleukin 6," The Journal of Biological Chemistry, vol. 277, no. 14, pp. 11802-11810, 2002.

[38] I. S. Singh, R. M. Viscardi, I. Kalvakolanu, S. Calderwood, and J. D. Hasday, "Inhibition of tumor necrosis factor-alpha transcription in macrophages exposed to febrile range temperature. A possible role for heat shock factor-1 as a negative transcriptional regulator," Journal of Biological Chemistry, vol. 275, no. 13, pp. 9841-9848, 2000.

[39] Y. Zhang, L. Huang, J. Zhang, D. Moskophidis, and N. F. Mivechi, "Targeted disruption ofhsf1 leads to lack of thermotolerance and defines tissue-specific regulation for stressinducible Hsp molecular chaperones," Journal of Cellular Biochemistry, vol. 86, no. 2, pp. 376-393, 2002.
[40] E. S. Christians, L. J. Yan, and I. J. Benjamin, "Heat shock factor 1 and heat shock proteins: critical partners in protection against acute cell injury," Critical Care Medicine, vol. 30, Suppl., pp. S43-S50, 2002.

[41] S. G. Ahn and D. J. Thiele, "Redox regulation of mammalian heat shock factor 1 is essential for Hsp gene activation and protection from stress," Genes \& Development, vol. 17, no. 4, pp. 516-528, 2003.

[42] P. A. Mercier, N. A. Winegarden, and J. T. Westwood, "Human heat shock factor 1 is predominantly a nuclear protein before and after heat stress," Journal of cell science, vol. 112, no. 16, pp. 2765-2774, 1999.

[43] M. Vujanac, A. Fenaroli, and V. Zimarino, "Constitutive nuclear import and stress-regulated nucleocytoplasmic shuttling of mammalian heat-shock factor 1," Traffic, vol. 6, no. 3, pp. 214-229, 2005. 